\title{
Novel Adenovirus type 5 vaccine platform induces cellular immunity against HIV-1 Gag, Pol, Nef despite the presence of Ad5 immunity
}

\author{
Elizabeth S. Gabitzsch ${ }^{1},{ }^{*}$, Younong $\mathrm{Xu}^{1}$, Lois H. Yoshida ${ }^{1}$, Joseph Balint ${ }^{1}$, Andrea \\ Amalfitano $^{2}$, and Frank R. Jones ${ }^{1}$ \\ ${ }^{1}$ Etubics Corporation, Seattle, WA \\ ${ }^{2}$ Department of Microbiology and Molecular Genetics, Michigan State University, East Lansing, MI
}

\begin{abstract}
Recombinant Adenovirus serotype 5 (Ad5) vectors have been used as vaccine platforms in numerous animal and human clinical studies. The immune response induced by Ad5 vaccines can be mitigated due to pre-existing Ad5 immunity. We report here the use of a novel Ad5 platform to induce cellular immune responses (CMI) in Ad5 hyper immunized mice. The effectiveness of the Ad5 [E1-, E2b-] vaccine platform was evaluated using HIV-1 Gag, Pol, and Nef as antigenic transgenes. Broad CMI was induced following vaccination with the HIV-1 expressing vectors in Ad5 naïve and Ad5 immunized mice. A mixture of the three vaccines induced CMI against each transgene product even in the presence of hyper Ad5 immunity.
\end{abstract}

\section{Keywords}

Adenovirus; vaccine; Ad5-immunity; HIV-1

\section{INTRODUCTION}

The level of cell mediated immunity (CMI) generated by infection or immunization correlates with protection against certain disease progression $(1,2)$. This may be the case because a robust CMI response suppresses viral replication as a result of infected cell destruction and subsequently may prevent the emergence of mutant viruses as well. Live viral vectors, including those based on Adenovirus (Ad), modified vaccinia virus Ankara (MVA), canarypox, vesicular stomatitis virus, herpes virus and others, have been reported to elicit protective CMI responses $(3,4)$. Among the cadre of vectors, Ad serotype 5 (Ad5) appears to have a number of advantages including the ability to induce mucosal immunity (5), direct infection of antigenpresenting dendritic cells $(6,7)$, induction of 5-10 fold greater CMI response than MVA and others $(8,9)$, higher protective effectiveness on a per-cytotoxic $\mathrm{T}$ cell basis $(10)$ and are well tolerated in human vaccinees $(10,11)$.

*Correspondence and reprint requests should be addressed to: Elizabeth S. Gabitzsch For regular mail: Etubics Corporation, 1124 Columbia Street, Suite 718 Seattle, WA 98104. Phone: (206) 838-5110; Fax: (206) 838-2978; beth@etubics.com.

Publisher's Disclaimer: This is a PDF file of an unedited manuscript that has been accepted for publication. As a service to our customers we are providing this early version of the manuscript. The manuscript will undergo copyediting, typesetting, and review of the resulting proof before it is published in its final citable form. Please note that during the production process errors may be discovered which could affect the content, and all legal disclaimers that apply to the journal pertain. 
Current generation Ad5 vectors utilized are deleted at the E1 and E3 or the E1, E3 and E4 regions (Ad5 [E1-]). Ad5 immunity has been shown to suppress the immunogenicity of Ad5 [E1-] vaccines in both preclinical studies and clinical trials $(8,12-14)$. This is a challenge due to the presence of Ad5 immunity in a high percentage of potential vaccinees due to natural infection (15). Investigators have proposed overcoming Ad5 immunity by using different $\mathrm{Ad}$ serotypes under the assumption that pre-existing vector specific immunity in the general population would not be as prevalent as it is to Ad5. An important issue with this technique is that once a vaccinee is immunized with an alternative serotype, a serotype specific immune response will be mounted. One cannot then administer a further re-immunization (boost) with that vaccine or use that viral backbone to vaccinate against a different antigen; therefore, terminating the future use of that alternative Ad vector in the vaccinee.

A uniquely modified Ad5 vector with deletion of the E2b region encoding viral DNA polymerase (pol) and the pre-terminal protein (pTP) genes (Ad5 [E1-, E2b-]) has been used to improve gene therapies $(16,17)$. This modification results in dramatic reduction of Ad5 late gene expression (16), which leads to increased quantity of stable transgene expression in a number of animal models $(18,19)$ and reduced acute toxicities as compared to Ad5 [E1-] vectors (20,21). Minimizing Ad5 viral protein expression may reduce the potential of Ad5 viral proteins from impacting host immune responses. We constructed Ad5 [E1-, E2b-] vectors expressing the HIV-1 Gag, Pol, and Nef genes as "target" antigens. Studies were performed to assess the propensity of the novel Ad5 [E1-, E2b-] vector to induce CMI responses in an Ad5 naïve and an Ad5 immune murine model. We found that the novel Ad5 [E1-, E2b-] viral vector can induce both a high frequency and a broad range of HIV-1 Gag, Pol, and Nef specific CMI responses following immunization despite the presence of Ad5 immunity.

\section{MATERIALS AND METHODS}

\subsection{Vaccine Vectors}

Using the HXB Gag, NL4-3 Pol and Nef genes (Genbank Accession \# K03455 for Gag, M19921 for Pol and Nef) derived from VRC-4401, VRC-4409, and VRC-4404, respectively (kindly provided by the Vaccine Research Center, NIAIDs, NIH), the Ad5 [E1-, E2b-]-gag, Ad5 [E1-, E2b-]-pol, and Ad5 [E1-, E2b-]-nef vectors were constructed, and vaccines were produced and purified according to previously described procedures (16).

\subsection{Animals}

Specific pathogen-free, female BALB/c mice (Jackson Laboratory, Bar Harbor, Maine) ages 6 to 8 weeks were housed in animal facilities at the Infectious Disease Research Institute (Seattle, Washington) and all procedures were conducted according to IACUC approved protocols.

\subsection{Immunization schedule}

Ad5 naïve mice ( $\mathrm{n}=5 /$ group) were immunized intradermally three times at two week intervals with $10^{10} \mathrm{VP}$ of Ad5 [E1-, E2b-]-gag, Ad5 [E1-, E2b-]-pol, or Ad5 [E1-, E2b-]-nef separately, or all three vaccines simultaneously for triad vaccination. To develop Ad5 immune mice, naïve mice were immunized twice with $10^{10} \mathrm{VP}$ of Ad5 [E1-]-null (an Ad5 [E1-] vector with no insert) at a two-week interval. As previously determined, two immunizations with $10^{10}$ VP Ad5 [E1-]-null induces an Ad5 neutralizing antibody titer of approximately 1:200. Fourteen days following the second Ad5 [E1-]-null immunization, mice were immunized three times at 14-day intervals with $10^{10} \mathrm{VP}$ of Ad5 [E1-, E2b-]-gag, Ad5 [E1-, E2b-]-pol, or Ad5 [E1-, E2b-]-nef. For the triad immunization protocol a mixture of $10^{10} \mathrm{VP}$ of each of the vaccines was used. Control mice were injected with injection buffer (20 mM HEPES with 3\% 
sucrose) alone. Fourteen days after the final immunization splenocytes from all mice were assessed by ELISpot assay for the secretion of IFN- $\gamma$ and IL-2.

\subsection{Adenovirus Neutralization Assay}

Dilutions of heat inactivated test sera in $100 \mu \mathrm{L}$ DMEM containing $10 \%$ fetal bovine serum were mixed with $4 \times 10^{10} \mathrm{VP}$ of Ad5 [E1-]-null and incubated for 60 minutes. Then the samples were added to microwells containing the HEK-293 cells at $2 \times 10^{3}$ cells/well and incubated for an additional 72 hours. An MTT reduction assay was used to quantify resultant cell killing, and endpoint Ad5 NAb titers were then determined.

\subsection{Enzyme-Linked Immunospot (ELISpot) Assay}

An ELISpot assay was used to detect HIV-1 Gag, Pol and Nef specific IFN- $\gamma$ and IL-2producing T cells from mouse splenocytes stimulated with Gag, Pol or Nef peptides (provided by the NIH AIDS Research and Reference Reagent Program). The ELISpot assay was run according to the manufacture's protocols (eBioscience, San Diego, CA). Splenocytes were used at a concentration of $2 \times 10^{5}$ cells/well and reported as the number of spots per $10^{6}$ cells per well. HIV-1 peptides were utilized at $0.2 \mu \mathrm{g}$ of each peptide/well. Cells stimulated with concanavalin A (ConA) at a concentration of $0.0625 \mu \mathrm{g} / \mathrm{well}$ served as a positive control. Colored spot-forming cells (SFC) were counted using an Immunospot ELISpot plate reader (Cellular Technology, Shaker Heights, $\mathrm{OH}$ ) and responses were considered to be positive if, 1) $50 \mathrm{SFC}$ were detected $/ 10^{6}$ cells after subtraction of the negative control and, 2) SFC were $\geq 2$-fold than those in the negative control wells. SFC quantities are reported as the number of observed spots per $10^{6}$ cells per well.

To map the breadth of the HIV-1 specific CMI response Gag, Pol and Nef pools consisting of 15 amino acid peptides with an 11-mer overlap were used to stimulate splenocytes. Peptide pools (PP) consisted of the following: 4 Gag PP with 31 peptides/pool spanning the entire gag protein and 5 Pol PP with 50 peptides/pool spanning the entire Pol protein.

\subsection{Statistical analysis}

Statistically significant differences in the mean CMI responses between groups of animals were determined by Student's $t$-test with a p-value of 0.05 or lower being considered significant, using GraphPad Prism ${ }^{\mathrm{R}}$ (GraphPad Software, Inc.).

\subsection{RESULTS}

\subsection{CMI responses to immunization in Ad5 naïve mice}

The immunogenicity of homologous vaccination in naïve mice was determined following three immunizations with Ad5 [E1-, E2b-]gag, Ad5 [E1-, E2b-]-pol, or Ad5 [E1-, E2b-]-nef. Splenocytes were collected 14 days after the final immunization and assessed by ELISpot to determine Gag, Pol, or Nef-specific IFN- $\gamma$ and IL-2 secretion. The IFN- $\gamma$ transgene-specific response was highest to $\mathrm{Pol}\left(6717 \pm 376 \mathrm{SFC} / 10^{6}\right.$ splenocytes) and was also robust to Gag (432 $\pm 157 \mathrm{SFC} / 10^{6}$ splenocytes) and Nef (578 $\pm 123 \mathrm{SFC} / 10^{6}$ splenocytes). Transgenespecific IL-2 secretion was detected at high levels in Pol-immunized mice $(1021 \pm 122 \mathrm{SFC} /$ $10^{6}$ splenocytes) and response to Gag-immunization ( $93 \pm 22 \mathrm{SFC} / 10^{6}$ splenocytes) and Nefimmunization ( $46 \pm 9 \mathrm{SFC} / 10^{6}$ splenocytes) was also recorded. These data confirm that Ad5 [E1-, E2b-]-gag, Ad5 [E1-, E2b-]-pol, and Ad5 [E1-, E2b-]-nef vaccines induce specific CMI responses in Ad5 naïve mice. 


\section{2 $\mathrm{CMI}$ responses to immunization in Ad5 immune mice}

HIV-1 transgene specific CMI responses following three immunizations with Ad5 [E1-, E2b -]-gag, Ad5 [E1-, E2b-]-pol, or Ad5 [E1-, E2b-]-nef was determined in Ad5 immune mice (mice were pre-immunized twice with Ad5 [E1-]-null). Fourteen days after the third vaccination splenocytes were assessed by ELISpot to determine the number of splenocytes secreting IFN- $\gamma$ and IL-2. Transgene-specific IFN- $\gamma$ secretion to Gag $(263 \pm 23$ SPC/10 splenocytes) and Pol (2406 $\pm 304 \mathrm{SFC} / 10^{6}$ splenocytes) as well as IL-2 specific secretion to Gag $\left(73 \pm 2 \mathrm{SFC} / 10^{6}\right.$ splenocytes $)$ and $\mathrm{Pol}\left(305 \pm 69 \mathrm{SFC} / 10^{6}\right.$ splenocytes were detected despite the presence of Ad5 immunity. Ad5 [E1-, E2b-]-nef also induced a measurable level of Nef specific CMI response for IFN- $\gamma$ secretion $\left(60 \pm 14 \mathrm{SFC} / 10^{6}\right.$ splenocytes), but not for IL-2.

\subsection{Breadth of CMI response in immunized mice}

The breadth of HIV-1 transgene specific CMI in both Ad5 naïve and Ad5 immune mice after three immunization with Ad5 [E1-, E2b-]gag or Ad5 [E1-, E2b-]-pol was determined. Fourteen days after the final vaccination splenocytes were stimulated with Gag or Pol PP, respectively. ELISpot analysis was used to determine IFN- $\gamma$ and IL-2 secretion by splenocytes therefore indicating recognition of that PP. Splenocytes from Ad5 naïve and Ad5 immune mice immunized Ad5 [E1-, E2b-]-gag recognized 2 out of the 4 Gag PP (Figure 3A,B). Splenocytes from naïve mice immunized with Ad5 [E1-, E2b-]-pol recognized 5 out of 5 PP and splenocytes from Ad5 immune mice recognized 3 out of 5 PP for IL-2 (Fig. 3C,D). These data indicate that the Ad5 [E1-, E2b-]-gag and Ad5 [E1-, E2b-]-pol induced a broad range of immune responses that recognized 7 out of $9 \mathrm{PP}$ in naïve mice and 5 out of $9 \mathrm{PP}$ in Ad5 immune mice.

\subsection{Triad vaccination with Ad5 [E1-, E2b-]-gag, Ad5 [E1-, E2b-]-pol, and Ad5 [E1-, E2b-]- nef}

The immune response elicited to the Ad5 [E1-, E2b-] HIV-1 vaccines when administered simultaneously to Ad5 naïve and Ad5 immune mice was evaluated. Mice were immunized three times at two week intervals with $10^{10}$ VP each of Ad5 [E1-, E2b-]-gag, Ad5 [E1-, E2b -]-pol, and Ad5 [E1-,E2b-,]-nef. As presented in Figure 5A, immunizations in naïve mice with the triad vaccine induced CMI against Gag $\left(472 \pm 90\right.$ SFC/ $10^{6}$ IFN- $\gamma$ secreting splenocytes and $93 \pm 17$ SFC/10 $10^{6}$ IL-2 secreting splenocytes), Pol (8194 \pm 305 SFC $/ 10^{6}$ IFN- $\gamma$ secreting splenocytes, $324 \pm 33 \mathrm{SFC} / 10^{6} \mathrm{IL}-2$ secreting splenocytes) and Nef (133 $\pm 28 \mathrm{SFC} / 10^{6} \mathrm{IFN}-\gamma$ secreting splenocytes, no detectable response for IL-2 secretion). Ad5 immune mice immunized with the triad vaccine demonstrated CMI against Gag, Pol and Nef despite the presence of high-titer Ad5 immunity (Figure 4B). These data indicate that the Ad5 [E1-, E2b -] HIV-1 triad vaccine can elicit transgene-specific immune responses in naïve and Ad5 immune mice when administered simultaneously.

\subsection{DISCUSSION}

As indicated by the Merck STEP trial, a vaccine comprised of Ad5 vectors must effectively deliver the transgenes despite the presence of Ad5 immunity in the vaccinee. Here we evaluated the efficacy of a novel Ad5 [E1-, E2b-,] vector expressing HIV-1 Gag, Pol and Nef proteins to induce CMI responses in both Ad5 naïve and Ad5 immune animals. We report that Ad5 [E1 -, E2b-]-gag, Ad5 [E1-, E2b-]-pol, and Ad5 [E1-, E2b-,]-nef vaccines induced INF- $\gamma$ secretion in not only Ad5 naïve but more importantly in Ad5 immune mice. Ad5 [E1-, E2b-] gag and Ad5 [E1-]-pol also induced IL-2 secretion in Ad5 naïve and Ad5 immune mice, demonstrating HIV-1 specific CMI in vaccinated mice. The propensity of the Ad5 [E1-, E2b -] vector to overcome the barrier of preexisting Ad5 immunity may be linked to dramatic reduction of expression of Ad5 late genes (16). Reduction of Ad5 late gene expression may 
induce a lower and slower memory immune response against the vector, allowing longer and stable vectored gene expression.

We also demonstrated that Ad5 [E1-, E2b-]-gag and Ad5 [E1-, E2b-]-pol induce a wide breadth CMI response. Previous reports showed that individuals with untreated chronic HIV-1 infection patients recognized significantly more protein subunits (median, 9 peptide pools out of total 100 pools) than individuals with treated acute infection (median, 4 peptide pools) screened with a total of 100 peptide pools spanning entire HIV-1 genome (22). Others have reported that there is a negative correlation between viral load and IFN- $\gamma$ production of HIV-1specific T cells using fewer epitopes or HIV-1 proteins (23-25). The broadening of the CMI repertoire may prevent viral escape during acute and early infection, thus resulting in a more efficacious control of viral replication. The capacity of Ad5 [E1-, E2b-] vaccine to induce broad CMI recognition of HIV-1 protein subunits will play an critical role in HIV-1 viral load control and may have important implication for HIV-1 vaccine design. To understand the mechanism of immune responses induced by the Ad5 [E1-, E2b-] vaccine platform, future studies, such as the investigation of detailed phenotypic, functional determinants and the "quality" of HIV-1-specific CMI responses are needed and are underway (26-28).

\subsection{References}

1. Jin X, Bauer DE, Tuttleton SE, et al. Dramatic rise in plasma viremia after CD8(+) T cell depletion in simian immunodeficiency virus-infected macaques. J Exp Med 1999;189:991-998. [PubMed: 10075982]

2. Schmitz JE, Kuroda MJ, Santra S, et al. Control of viremia in simian immunodeficiency virus infection by CD8+ lymphocytes. Science 1999;283:857-860. [PubMed: 9933172]

3. Gómez-Román VR, Robert-Guroff M. Adenoviruses as vectors for HIV vaccines. AIDS Rev 2003;5:178-185. [PubMed: 14598567]

4. Robinson HL. New hope for an AIDS vaccine. Nat Rev Immunol 2002;2:239-250. [PubMed: 12001995]

5. Gallichan WS, Rosenthal KL. Long-lived cytotoxic T lymphocyte memory in mucosal tissues after mucosal but not systemic immunization. J Exp Med 1996;184:1879-1890. [PubMed: 8920875]

6. Jooss K, Yang Y, Fisher KJ, Wilson JM. Transduction of dendritic cells by DNA viral vectors directs the immune response to transgene products in muscle fibers. J Virol 1998;72:4212-4223. [PubMed: 9557710]

7. Kirk CJ, Mulé JJ. Gene-modified dendritic cells for use in tumor vaccines. Hum Gene Ther 2000;11:797-806. [PubMed: 10779157]

8. Casimiro DR, Chen L, Fu TM, et al. Comparative immunogenicity in rhesus monkeys of DNA plasmid, recombinant vaccinia virus, and replication-defective adenovirus vectors expressing a human immunodeficiency virus type 1 gag gene. J Virol 2003;77:6305-6313. [PubMed: 12743287]

9. Yang ZY, Wyatt LS, Kong WP, Moodie Z, Moss B, Nabel GJ. Overcoming immunity to a viral vaccine by DNA priming before vector boosting. J Virol 2003;77:799-803. [PubMed: 12477888]

10. Shiver JW, Fu TM, Chen L, et al. Replication-incompetent adenoviral vaccine vector elicits effective anti-immunodeficiency-virus immunity. Nature 2002;415:331-335. [PubMed: 11797011]

11. Catanzaro AT, Koup RA, Roederer M, et al. Phase 1 safety and immunogenicity evaluation of a multiclade HIV-1 candidate vaccine delivered by a replication-defective recombinant adenovirus vector. J Infect Dis 2006;194:1638-1649. [PubMed: 17109335]

12. Fitzgerald JC, Gao GP, Reyes-Sandoval A, et al. A simian replication-defective adenoviral recombinant vaccine to HIV-1 gag. J Immunol 2003;170(3):1416-22. [PubMed: 12538702]

13. Shiver JW, Emini EA. Recent advances in the development of HIV-1 vaccines using replicationincompetent adenovirus vectors. Annu Rev Med 2004;55:355-372. [PubMed: 14746526]

14. Yang ZY, Wyatt LS, Kong WP, Moodie Z, Moss B, Nabel GJ. Overcoming immunity to a viral vaccine by DNA priming before vector boosting. J Virol 2003;77:799-803. [PubMed: 12477888] 
15. Xiang Z, Gao G, Reyes-Sandoval A, et al. Novel, chimpanzee serotype 68-based adenoviral vaccine carrier for induction of antibodies to a transgene product. J Virol 2002;76:2667-2675. [PubMed: 11861833]

16. Amalfitano A, Hauser MA, Hu H, Serra D, Begy CR, Chamberlain JS. Production and characterization of improved adenovirus vectors with the E1, E2b, and E3 genes deleted. J Virol 1998;72:926-933. [PubMed: 9444984]

17. Hauser MA, Amalfitano A, Kumar-Singh R, Hauschka SD, Chamberlain JS. Improved adenoviral vectors for gene therapy of Duchenne muscular dystrophy. Neuromuscul Disord 1997;7:277-283. [PubMed: 9267841]

18. Hu H, Serra D, Amalfitano A. Persistence of an [E1-, polymerase-] adenovirus vector despite transduction of a neoantigen into immune-competent mice. Hum Gene Ther 1999;10:355-364. [PubMed: 10048388]

19. Hodges BL, Serra D, Hu H, Begy CA, Chamberlain JS, Amalfitano A. Multiply deleted [E1, polymerase-, and pTP-] adenovirus vector persists despite deletion of the preterminal protein. J Gene Med 2000;2:250-259. [PubMed: 10953916]

20. Everett RS, Hodges BL, Ding EY, Xu F, Serra D, Amalfitano A. Liver toxicities typically induced by first-generation adenoviral vectors can be reduced by use of E1, E2b-deleted adenoviral vectors. Hum Gene Ther 2003;14:1715-1726. [PubMed: 14670123]

21. Schaack J. Induction and inhibition of innate inflammatory responses by adenovirus early region proteins. Viral Immunol 2005;18:79-88. [PubMed: 15802954]

22. Addo MM, Yu XG, Rathod A, et al. Comprehensive epitope analysis of human immunodeficiency virus type 1 (HIV-1)-specific T-cell responses directed against the entire expressed HIV-1 genome demonstrate broadly directed responses, but no correlation to viral load. J Virol 2003;77:2081-2092. [PubMed: 12525643]

23. Ogg GS, Jin X, Bonhoeffer S, et al. Quantitation of HIV-1-specific cytotoxic T lymphocytes and plasma load of viral RNA. Science 1998;279:2103-2106. [PubMed: 9516110]

24. Edwards BH, Bansal A, Sabbaj S, Bakari J, Mulligan MJ, Goepfert PA. Magnitude of functional CD8 + T-cell responses to the gag protein of human immunodeficiency virus type 1 correlates inversely with viral load in plasma. J Virol 2002;76:2298-2305. [PubMed: 11836408]

25. Appay V, Dunbar PR, Callan M, et al. Memory CD8+ T cells vary in differentiation phenotype in different persistent virus infections. Nat Med 2002;8:379-385. [PubMed: 11927944]

26. Champagne P, Ogg GS, King AS, et al. Skewed maturation of memory HIV-specific CD8 T lymphocytes. Nature 2001;410:106-111. [PubMed: 11242051]

27. Rowland-Jones SL, Pinheiro S, Kaul R, et al. How important is the 'quality' of the cytotoxic T lymphocyte (CTL) response in protection against HIV infection? Immunol Lett 2001;79:15-20. [PubMed: 11595285]

28. Schmitz JE, Kuroda MJ, Santra S, et al. Control of viremia in simian immunodeficiency virus infection by CD8+ lymphocytes. Science 1999;283:857-860. [PubMed: 9933172] 

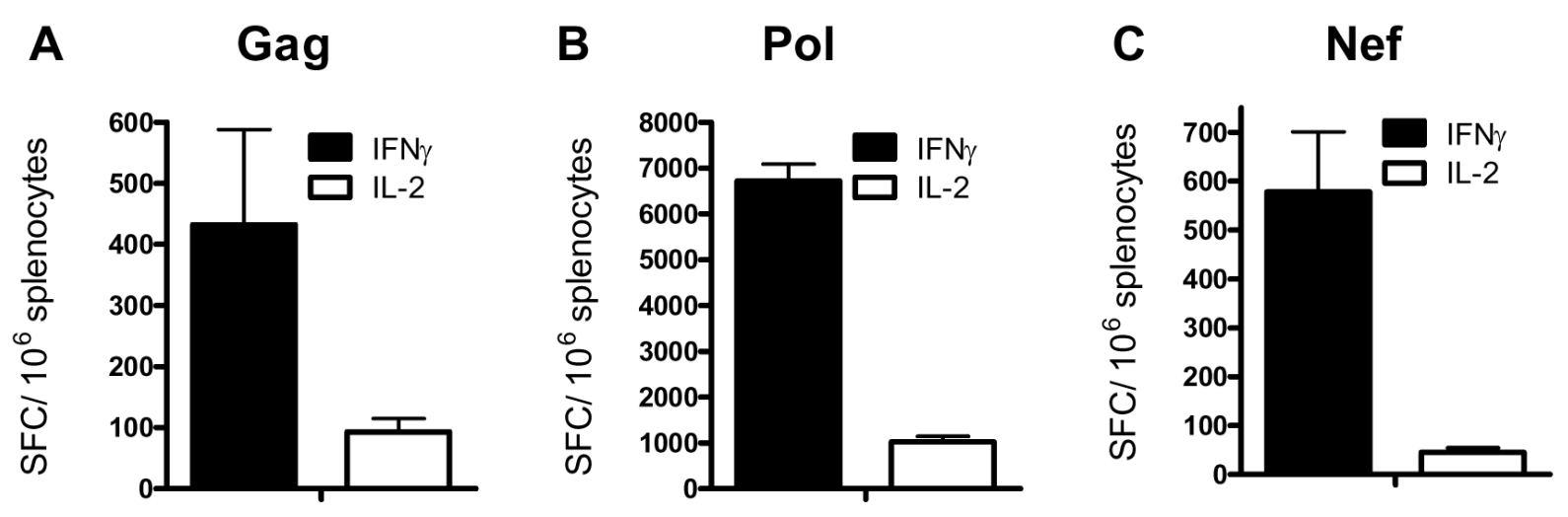

Figure 1. CMI responses to immunizations in Ad5 naïve mice

Naïve BALB/c mice ( $\mathrm{n}=5$ /group) were immunized three times with $10^{10}$ VP of Ad5 [E1-, E2b -]-gag (A), Ad5 [E1-, E2b-]-pol (B) or Ad5 [E1-, E2b-]-nef (C). Fourteen days after the final immunization splenocytes from the mice were assessed for IFN- $\gamma$ or IL-2 secreting splenocytes by ELISpot analysis. Vertical bars indicate the standard error of the mean (SEM). 


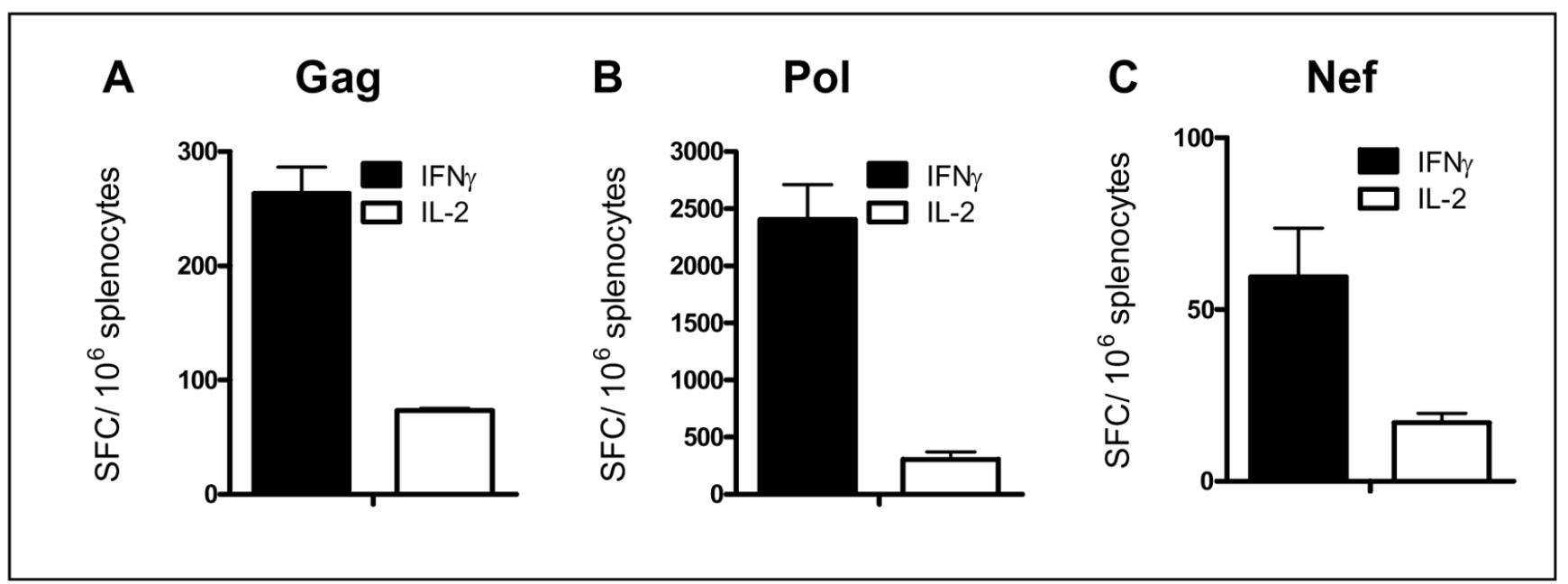

Figure 2. CMI response to immunizations in Ad5 immune mice

Naïve BALB/c mice were pre-immunized 2 times with $10^{10}$ VP Ad5 [E1-]-null and then immunized three times with $10^{10}$ VP Ad5 [E1-]-gag (A), Ad5 [E1-, E2b-]-pol (B) or Ad5 [E1-, E2b-]-nef (C). Splenocytes were collected 14 days after the final immunization and assessed for IFN- $\gamma$ or IL-2 secreting splenocytes by ELISpot. Vertical bars indicate the SEM. 
A Ad naive

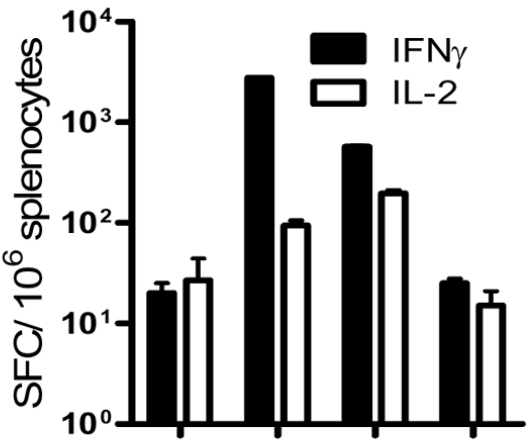

Figure 3. Breadth of transgene specific cellular immunity in Ad5 naïve and hyper immune mice after immunizations with Ad5 [E1-, E2b-]-gag or Ad5 [E1-, E2b-]-pol

Naive or Ad5 immune mice were immunized three times with Ad5 [E1-, E2b-]-gag or Ad5

$[\mathrm{E} 1-, \mathrm{E} 2 \mathrm{~b}-]-$ pol. Splenocytes were collected 14 days after the final immunization and stimulated with Gag (A, B) or Pol (C, D) peptide pools. Cells were assessed for IFN- $\gamma$ or IL-2 secreting splenocytes by ELISpot. Vertical bars indicate the SEM. 


\section{A Ad naive}

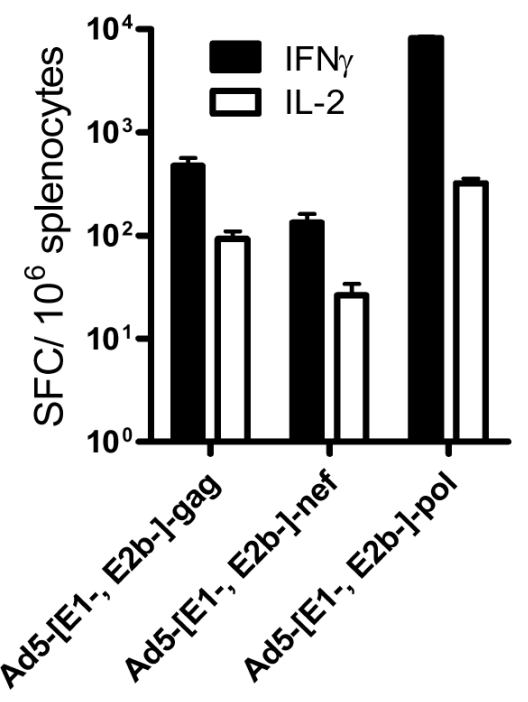

\section{B Ad immune}

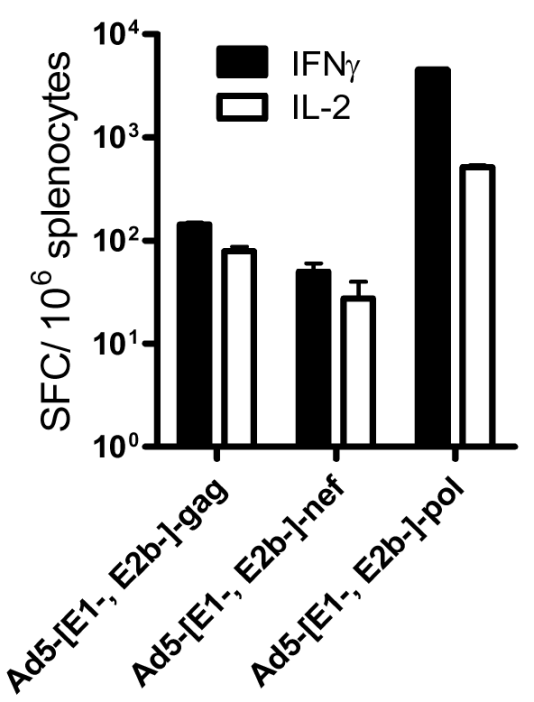

Figure 4. Triad vaccination with Ad5 [E1-, E2b-]-gag, Ad5 [E1-, E2b-]-pol, and Ad5 [E1-, E2b -]-nef

Naïve (A) or Ad5 immune (B) BALB/c mice (n=5/group) were immunized three times with $10^{10} \mathrm{VP}$ of Ad5 [E1-, E2b-]-gag (A), Ad5 [E1-, E2b-]-pol (B) and Ad5 [E1-, E2b-]-nef

(C). Fourteen days after the final immunization splenocytes from the mice were assessed for IFN- $\gamma$ or IL-2 secreting splenocytes by ELISpot. Vertical bars indicate the standard error of the mean (SEM). 